\title{
Author Correction: Proteomic analysis of lymphoblastoid cell lines from schizophrenic patients
}

Akira Yoshimi $\mathbb{0}^{1,2,3}$, Shinnosuke Yamada ${ }^{1,2}$, Shohko Kunimoto ${ }^{3}$, Branko Aleksic $\mathbb{D}^{3}$, Akihiro Hirakawa ${ }^{4}$, Mitsuki Ohashi', Yurie Matsumoto ${ }^{1,3}$, Kazuhiro Hada2, Norimichi Itoh2, Yuko Arioka ${ }^{3,5}$, Hiroki Kimura 3,6, Itaru Kushima 3,6,7, Yukako Nakamura ${ }^{3}$, Tomoko Shiino $\mathbb{B}^{3,8}$, Daisuke Mori $\mathbb{0}^{3,9}$, Satoshi Tanaka $\mathbb{E}^{6}$, Shuko Hamada ${ }^{3}$, Yukihiro Noda ${ }^{1,2,3}$, Taku Nagai ${ }^{2}$, Kiyofumi Yamada ${ }^{2}$ and Norio Ozaki $\mathbb{1}^{3,6}$

\section{Correction to: Translational Psychiatry} https://doi.org/10.1038/s41398-019-0461-2 Published online 22 April 2019

The original Article required a few updates; one co-author name, which was given as Hiroki Kiumura, has been updated to Hiroki Kimura. Furthermore, supplementary information has been updated, and grant numbers have been added. These updates have been made to both the PDF and HTML versions of this Article.

Published online: 03 May 2019

\footnotetext{
Correspondence: Branko Aleksic (branko@med.nagoya-u.ac.jp)

${ }^{1}$ Division of Clinical Sciences and Neuropsychopharmacology, Faculty and Graduate School of Pharmacy, Meijo University, Nagoya 468-8503, Japan

2Department of Neuropsychopharmacology and Hospital Pharmacy, Nagoya University Graduate School of Medicine, Nagoya 466-8550, Japan

${ }^{3}$ Department of Psychiatry, Nagoya University Graduate School of Medicine, Nagoya 466-8550, Japan

${ }^{4}$ Department of Biostatistics and Bioinformatics, Graduate School of Medicine, University of Tokyo, Tokyo 113-0033, Japan

${ }^{5}$ Center for Advanced Medicine and Clinical Research, Nagoya University Hospital, Nagoya 466-8550, Japan

${ }^{6}$ Department of Psychiatry, Nagoya University Hospital, Nagoya 466-8550, Japan

${ }^{7}$ Institute for Advanced Research, Nagoya University, Nagoya 464-8601, Japan ${ }^{8}$ Department of Pathology of Mental Diseases, National Institute of Mental Health, National Center of Neurology and Psychiatry, Tokyo 187-8553, Japan ${ }^{9}$ Brain and Mind Research Center, Nagoya University, Nagoya 466-8550, Japan These authors contributed equally: Akira Yoshimi, Shinnosuke Yamada, Shohko Kunimoto
} changes were made. The images or other third party material in this article are included in the article's Creative Commons license, unless indicated otherwise in a credit line to the material. If material is not included in the article's Creative Commons license and your intended use is not permitted by statutory regulation or exceeds the permitted use, you will need to obtain permission directly from the copyright holder. To view a copy of this license, visit http://creativecommons.org/licenses/by/4.0/. 